\title{
AN ALGEBRAIC SOLUTION TO ROTATION RECOVERY IN HARDI FROM CORRESPONDENCES OF ORIENTATION DISTRIBUTION FUNCTIONS
}

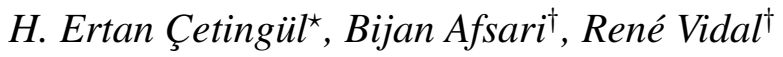 \\ ^Imaging and Visualization, Siemens Corporate Research \& Technology, Princeton, NJ, USA \\ ${ }^{\dagger}$ Center for Imaging Science, Johns Hopkins University, Baltimore, MD, USA
}

\begin{abstract}
We consider the problem of aligning high angular resolution diffusion images characterized by orientation distribution functions (ODFs). We cast this problem as an optimization problem where we seek the rotation that aligns the source and target ODFs. This rotation induces a linear transformation of the spherical harmonic coefficients of the ODFs, which can be parametrized by the rotation Euler angles. We propose an algebraic approach to estimate this transformation from a number of ODF correspondences. We evaluate the proposed method on synthetic ODFs as well as on a diffusion MR phantom dataset.
\end{abstract}

Index Terms - image matching, optimization, irreducible representation, harmonic analysis, diffusion magnetic resonance imaging.

\section{INTRODUCTION}

High angular resolution diffusion imaging (HARDI) is a diffusion MRI technique that can be used to infer the tissue microstructure in vivo [1]. This requires the reconstruction of the orientation distribution function (ODF), a non-parametric probability density function (PDF) describing the anisotropy of water diffusion at a location of interest. The ODF model offers improved accuracy in resolving intravoxel complexities over the diffusion tensor (DT) model [2], currently the de facto standard for neuroimaging.

The problem of aligning ODFs can be considered as part of the more general problem of spatially normalizing HARDI data. Tools for normalizing human brain HARDI datasets are essential to investigate brain development and the onset and progression of neurological disorders by allowing one to quantify intra- and inter-subject differences in longitudinal and population studies. Recent works addressing the problem of normalization include but not limited to [3, 4, 5, 6, 7]. The method in [3] uses the spherical harmonic $(\mathrm{SH})$ representation of ODFs for extracting spatial features that are hierarchically updated by increasing the harmonic degree. The ODF images are registered by using these features in a forward-backward-consistent soft correspondence matching scheme. [4] performs registration of ODF images by using the $\mathrm{SH}$ band energies as rotationally invariant features in a multi-channel diffeomorphic demons algorithm. [5, 6] use the SH coefficients of the ODFs as features to find the diffeomorphism between the source and target images. [7] incorporates the Riemannian metric of ODFs for quantifying the similarity between the two images into a variational problem defined under the large deformation diffeomorphic metric mapping (LDDMM) framework.

In this paper, we approach the problem of aligning HARDI data from a different perspective. Rather than extending off-the-shelf registration techniques from intensity values to ODFs, we leverage the

H. Ertan Çetingül performed this work while at JHU. mathematical relationship between the spherical harmonic representations of the source and target ODFs to algebraically compute the optimal rotation aligning these ODFs. Under the assumption that the rotation is parameterized by the Euler angles in $z y z$ convention, the rotation of an ODF induces a linear transformation of the SH coefficients of that ODF parameterized by the same angles. [8] provided an iterative approach to recover the rotation, i.e., the Euler angles, from a pair of spherical images represented via $\mathrm{SH}$ coefficients. While a single correspondence is sufficient to estimate the rotation in our problem as well, erroneous correspondences and/or image noise will make the solution unreliable. In addition, [8] requires a "very good" initialization and hence, it can only be used for refining an existing solution, as reported by the authors themselves in [9]. Our main contribution is to propose an algorithm to estimate the rotation from multiple ODF correspondences by computing its Euler angles in a sequential way to avoid local optima. As a consequence, our method is less sensitive to initialization. Experiments on synthetic data evaluate the sensitivity of our approach to angular perturbations, image noise, and the number of correspondences. Experiments on phantom data demonstrate the validity of the method to recover rigid deformations.

\section{SPHERICAL HARMONIC REPRESENTATION OF ODFS}

Consider the spherical harmonic function $Y_{l}^{m}: \mathbb{S}^{2} \mapsto \mathbb{C}$ of degree $l$ and order $m$ of the form

$$
Y_{l}^{m}(\theta, \phi)=\sqrt{\frac{2 l+1}{4 \pi} \frac{(l-m) !}{(l+m) !}} P_{l}^{m}(\cos \theta) e^{i m \phi},
$$

where $P_{l}^{m}$ is the associated Legendre polynomial of the same degree and order, and $\theta \in[0, \pi]$ and $\phi \in[0,2 \pi)$ are the polar and azimuthal angles, respectively, representing the direction $(\theta, \phi)$ in spherical coordinates. Any complex-valued function defined on the sphere $\mathbb{S}^{2}$ can be expressed as a combination of spherical harmonics. However, due to the physics of the diffusion phenomenon, the ODF, $p$, is assumed to be real and antipodally symmetric. Thus, the $\mathrm{SH}$ representation of the ODF uses a modified basis that is also real and symmetric. More precisely, the value of the ODF at $(\theta, \phi)$ is approximated as

$$
p(\theta, \phi) \approx \sum_{l=0}^{L} \sum_{m=-l}^{l} d_{m}^{l} Y_{l m}(\theta, \phi) .
$$

Here, $\left\{d_{m}^{l}\right\}$ are the real SH coefficients and $\left\{Y_{l m}\right\}$ is the modified basis of degree $L$ containing $R=\frac{(L+1)(L+2)}{2}$ functions of the form

$$
Y_{l m}= \begin{cases}\sqrt{2} \operatorname{Re}\left\{Y_{l}^{|m|}\right\} & \text { if }-l \leq m<0, \\ Y_{l}^{m} & \text { if } m=0, \\ \sqrt{2}(-1)^{m+1} \operatorname{Im}\left\{Y_{l}^{m}\right\} & \text { if } 0<m \leq l,\end{cases}
$$


where $l=0,2,4, \ldots, L, m=-l, \ldots, l$, and $\operatorname{Re}\{\cdot\}$ and $\operatorname{Im}\{\cdot\}$ are the real and imaginary parts, respectively [10].

Similar to the complex SH functions $\left\{Y_{m}^{l}\right\}$, the modified SH functions $\left\{Y_{l m}\right\}$ form an orthonormal basis for $\mathbb{R}^{R}$. Therefore, the $\ell_{2}$ distance between two ODFs $p_{1}$ and $p_{2}$ can be found by computing the $\ell_{2}$ distance between their SH coefficient vectors $\mathbf{d}_{1}$ and $\mathbf{d}_{2}$, i.e.,

$$
\operatorname{dist}\left(p_{1}, p_{2}\right)=\left\|\mathbf{d}_{1}-\mathbf{d}_{2}\right\|_{2}=\left(\sum_{\substack{l=0 \\ l \text { even }}}^{L} \sum_{m=-l}^{l}\left|d_{m ; 1}^{l}-d_{m ; 2}^{l}\right|^{2}\right)^{\frac{1}{2}} .
$$

Finally, since (3) induces a linear transformation between $\left\{Y_{m}^{l}\right\}$ and $\left\{Y_{l m}\right\}$, one can convert the vector of real SH coefficients $\mathbf{d}=$ $\left[d_{0}^{0}, \ldots, d_{L}^{L}\right]^{\top}$ to that of complex SH coefficients $\hat{\mathbf{d}}=\left[\hat{d}_{0}^{0}, \ldots, \hat{d}_{L}^{L}\right]^{\top}$, and (4) also holds for the complex coefficients $\left\{\hat{d}_{m}^{l}\right\}$.

\section{ODF ROTATION THROUGH SPHERICAL HARMONICS}

Suppose that $\mathbf{R}$ is an element of the rotation group

$$
\mathrm{SO}(3)=\left\{\mathbf{R} \in \mathbb{R}^{3 \times 3} \mid \mathbf{R}^{\top}=\mathbf{R}^{-1}, \operatorname{det}(\mathbf{R})=1\right\}
$$

parameterized with the Euler angles $\alpha, \gamma \in[0,2 \pi), \beta \in[0, \pi]$ in $z y z$ convention, i.e., $\mathbf{R} \equiv \mathbf{R}(\alpha, \beta, \gamma)=\mathbf{R}_{z}(\gamma) \mathbf{R}_{y}(\beta) \mathbf{R}_{z}(\alpha)$, where $\mathbf{R}_{z}$ and $\mathbf{R}_{y}$ represent rotations about the $z$ and $y$ axes, respectively. Through the action of $\mathrm{SO}(3)$, denoted by "O", $\mathbf{R}$ induces a linear transformation of the complex SH coefficients $\left\{\hat{d}_{m}^{l}\right\}$ of the ODF $p$, which can also be obtained from the its real SH coefficients $\left\{d_{m}^{l}\right\}$. In particular, each coefficient $\hat{f}_{m}^{l}$ of the rotated ODF $\mathbf{R} \circ p$ can be written as a linear combination of the coefficients $\left\{\hat{d}_{m}^{l}\right\}$ of $p$ as

$$
\hat{f}_{m}^{l}=\sum_{k=-l}^{l} \hat{d}_{k}^{l} A_{k, m}^{l}(\mathbf{R})
$$

In this expression, $A_{k, m}^{l}$ is of the form

$$
A_{k, m}^{l}(\mathbf{R}(\alpha, \beta, \gamma))=e^{-i k \gamma} P_{k, m}^{l}(\cos \beta) e^{-i m \alpha},
$$

where $P_{k, m}^{l}$ are the generalizations of the associated Legendre polynomials, which can be computed efficiently via Jacobi polynomials and recurrence relations [11]. It is also shown in [11] that $A_{k, m}^{l}(\mathbf{R})$ are the matrix elements of the irreducible unitary representation of $\mathbf{R} \in \mathrm{SO}(3)$. This means that, for each $l$, the matrix with entries $A_{k, m}^{l}$ is unitary. These entries are also related to the elements of the $d$-small Wigner matrix [12]. To simplify the notation, we define the matrix $\mathbf{A}^{l} \in \mathbb{C}^{(2 l+1) \times(2 l+1)}$, whose $(k, m)$ entry is given by

$$
\left[\mathbf{A}^{l}\right]_{k, m}=A_{g(k, l), g(m, l)}^{l},
$$

where $g(k, l)=k+l+1, l=0,2, \ldots, L$, and $|k| \leq l$. If we write the vectors $\hat{\mathbf{f}}$ and $\hat{\mathbf{d}}$ of SH coefficients by concatenating the bands as $\hat{\mathbf{f}}=\left[\hat{\mathbf{f}}^{0},\left(\hat{\mathbf{f}}^{2}\right)^{\top}, \ldots,\left(\hat{\mathbf{f}}^{L}\right)^{\top}\right]^{\top}$ and $\hat{\mathbf{d}}=\left[\hat{\mathbf{d}}^{0},\left(\hat{\mathbf{d}}^{2}\right)^{\top}, \ldots,\left(\hat{\mathbf{d}}^{L}\right)^{\top}\right]^{\top}$, the expression in (6) can be written as $\hat{\mathbf{f}}=\mathbf{A}^{\top} \hat{\mathbf{d}}$, where $\mathbf{A}$ is a blockdiagonal matrix whose blocks $\left\{\mathbf{A}^{l}\right\}$ are unitary, i.e., $\left(\mathbf{A}^{l}\right)^{*} \mathbf{A}^{l}=$ $\mathbf{I}_{(2 l+1) \times(2 l+1)}$.

\section{ROTATION ESTIMATION FROM MULTIPLE ODF CORRESPONDENCES}

Our formulation utilizes a number of correspondences between two ODF images to estimate the matrix $\mathbf{A}$, whose entries (7) are further used for computing the Euler angles of $\mathbf{R}, \beta$ and $\{\alpha, \gamma\}$, in that order. Let $\left\{\left(p_{1 n}, p_{2 n}\right)\right\}_{n=1}^{N}$ be $N$ ODF correspondences, which can be manually provided by a medical expert or automatically found via feature extraction and matching. We assume that the ODFs are expressed in terms of their complex SH coefficients $\left\{\left(\hat{\mathbf{d}}_{1 n}, \hat{\mathbf{d}}_{2 n}\right)\right\}_{n=1}^{N}$ and seek to minimize $\sum_{n} \operatorname{dist}^{2}\left(p_{1 n}, \mathbf{R} \circ p_{2 n}\right)$, i.e.,

$$
E(\mathbf{A})=\frac{1}{N} \sum_{n=1}^{N}\left\|\hat{\mathbf{d}}_{1 n}-\mathbf{A}^{\top} \hat{\mathbf{d}}_{2 n}\right\|_{2}^{2}
$$

Since $\mathbf{A}$ is a function of $(\alpha, \beta, \gamma)$, we could directly minimize $E(\mathbf{A})$ over $(\alpha, \beta, \gamma)$ using nonlinear optimization. However, this strategy would be prone to local minima. We thus seek an alternative approach where we first optimize over $\mathbf{A}$ and then obtain $(\alpha, \beta, \gamma)$ from $\mathbf{A}$. The advantage of this approach is that the optimal solution for $\mathbf{A}$ is a global minimizer and can be obtained in closed form. In what follows, we consider the SH basis of degree $L=4 \Rightarrow R=15$.

Solving for $\mathbf{A}$. Notice that $\mathbf{A}$ is block-diagonal and $\mathbf{A}^{0}=1$. Thus, we can solve (9) for each even degree $l>0$ as follows:

$$
\min _{\mathbf{A}^{l}} E\left(\mathbf{A}^{l}\right)=\min _{\mathbf{A}^{l}} \frac{1}{N} \sum_{n=1}^{N}\left\|\hat{\mathbf{d}}_{1 n}^{l}-\left(\mathbf{A}^{l}\right)^{\top} \hat{\mathbf{d}}_{2 n}^{l}\right\|_{2}^{2}
$$

such that $\left(\mathbf{A}^{l}\right)^{*} \mathbf{A}^{l}=\mathbf{I}_{(2 l+1) \times(2 l+1)}$. Since $\mathbf{A}^{l}$ is unitary, minimizing $E\left(\mathbf{A}^{l}\right)$ is equivalent to maximizing $\operatorname{trace}\left(\sum_{n} \hat{\mathbf{d}}_{2 n}^{l}\left(\hat{\mathbf{d}}_{1 n}^{l}\right)^{*}\left(\mathbf{A}^{l}\right)^{\top}\right)$. Let $\mathbf{D}^{l}=\sum_{n} \hat{\mathbf{d}}_{2 n}^{l}\left(\hat{\mathbf{d}}_{1 n}^{l}\right)^{*}$, then $\tilde{\mathbf{A}}^{l}=\left(\mathbf{U V}^{*}\right)^{\top}$ is a solution to this problem, where $\mathbf{U} \mathbf{\Sigma} \mathbf{V}^{*}$ is the singular value decomposition of $\mathbf{D}^{l}$, and it is the unique solution if $\mathbf{D}^{l}$ is full-rank [13]. The matrix $\mathbf{D}^{l}$ is, in general, a full-rank matrix, unless $N$ is smaller than $2 l+1$ or many of the ODF correspondences are duplicate of each other. Both cases are very unlikely in the case of real data.

Solving for $\beta$ and $\{\alpha, \gamma\}$. Once the irreducible unitary representations $\left\{\tilde{\mathbf{A}}^{l}\right\}_{l \in\{2,4\}}$ are obtained, $\beta$ is estimated by setting $k=m=0$ in (7) and solving the resulting set of equations

$$
\left\{\tilde{A}_{l+1, l+1}^{l}=P_{0,0}^{l}(\cos \beta)\right\}_{l \in\{2,4\}} .
$$

In particular, by using $P_{0,0}^{1}(\cos \beta)=\cos \beta$ and the recurrence relations between $P_{k, m}^{l-1}, P_{k, m}^{l}$, and $P_{k, m}^{l+1}$, we obtain two equations

$$
\begin{aligned}
& 2 \tilde{A}_{3,3}^{2}=3 \cos ^{2} \beta-1, \\
& 8 \tilde{A}_{5,5}^{4}=35 \cos ^{4} \beta-30 \cos ^{2} \beta+3,
\end{aligned}
$$

which are solved for $\beta \in\left[0, \frac{\pi}{2}\right] .{ }^{1}$ Once a solution $\tilde{\beta}$ is computed, we subsequently estimate the remaining angles $\alpha$ and $\gamma$ by solving

$$
\min _{\alpha, \gamma} \sum_{\substack{l=2 \\ l \text { even }}}^{4} \sum_{k=-l}^{l} \sum_{m=-l}^{l}\left|\tilde{A}_{k, m}^{l}-e^{-i k \gamma} P_{k, m}^{l}(\cos \tilde{\beta}) e^{-i m \alpha}\right|^{2} .
$$

For this purpose, we employ a well-known quasi-Newton method called Broyden's method, which requires a good initialization to avoid potential local minima. Therefore, we first solve

$$
\begin{gathered}
\left|\tilde{A}_{0, m}^{2}-e^{-i m \alpha} P_{0, m}^{2}(\cos \tilde{\beta})\right|=0, m \in\{-2,2\}, \\
\left|\tilde{A}_{k, 0}^{2}-e^{-i k \gamma} P_{k, 0}^{2}(\cos \tilde{\beta})\right|=0, k \in\{-2,2\},
\end{gathered}
$$

for $\alpha$ and $\gamma$, respectively, and initialize our solver with the averages of these solutions. The resulting estimates $\{\tilde{\alpha}, \tilde{\gamma}\}$ are restricted to be in $[0, \pi)$ due to the antipodal symmetry of the ODFs. ${ }^{2}$ This last step finalizes the estimation of the rotation as $\tilde{\mathbf{R}}=\mathbf{R}(\tilde{\alpha}, \tilde{\beta}, \tilde{\gamma})$.

\footnotetext{
${ }^{1}$ In reality, it is seldom that $\beta>\frac{\pi}{2}$, hence we discard the root in $\left(\frac{\pi}{2}, \pi\right]$.

${ }^{2}$ Notice that if $\tilde{\beta} \approx 0$, we can take $\gamma=0$ and solve (14) for $\alpha$.
} 


\section{METHOD VALIDATION}

\subsection{Experiments on Synthetic Data}

We first study the sensitivity of our method to the amount of angular perturbations, the level of image measurement noise, and the number of ODF correspondences through experiment on synthetic data. We generate the synthetic data using the multi-tensor model, where the HARDI signal at a gradient direction $\mathbf{g}$ is a convex combination of functions of the form $e^{-b \mathbf{g}^{\top} \mathbf{T}_{r} \mathbf{g}}$. Here, $\mathbf{T}_{r}$ denotes the $r$-th tensor and we set $b=3,000 \mathrm{~s} / \mathrm{mm}^{2}$. We simulate the noise-free signal at 81 gradient directions and reconstruct 100 ODFs using the method proposed in [14]. We rotate each ODF with known parameters $\alpha, \gamma \in$ $\left\{0, \frac{\pi}{6}, \ldots, \frac{5 \pi}{6}\right\}, \beta \in\left\{0, \frac{\pi}{6}, \ldots, \frac{\pi}{2}\right\}$ to obtain its corresponding ODF, and estimate the rotation as described in Section 4. In this experiment, we particularly consider the following test scenarios:

Noisy rotations. We use the ODFs estimated from the noise-free signals and generate the corresponding ODFs by applying noisy rotations. More specifically, the Euler angles are corrupted by additive Gaussian noise with zero mean and varying standard deviation $\sigma_{\text {rot }} \in\left\{\frac{\pi}{180}, \frac{\pi}{90}, \frac{\pi}{45}, \frac{\pi}{18}\right\}$. Fig. 1 (top row) illustrates, for a randomly selected ODF, the generation of the corresponding ODFs. Notice that the higher the noise, the more the direction at which an ODF attains its peak deviates from the correct one (indicated in black).

Noisy measurements. We consider both the noise-free and noisy HARDI signals, which are obtained by adding complex Gaussian noise with zero mean and standard deviation $\sigma=1 / \zeta, \zeta$ being the signal-to-noise ratio (SNR). We form a correspondence by taking two ODFs, one reconstructed from the noise-free signal and the other one reconstructed from the noisy signals (SNR $\in\{40,30,20,10,5\}$ ), and then rotating the second ODF. We aim to quantify how differences in the signal quality, e.g., due to different acquisition settings and/or using raw diffusion weighted images, affect the estimation. Fig. 1 (bottom row) shows the corresponding ODFs for different SNRs.

We repeat these scenarios for $N \in\{20,40,60,80,100\}$ to investigate the effect of the number of correspondences. Tables 1 and 2 show the average of the angular discrepancies $\delta_{\rho}=|\tilde{\rho}-\rho|$ between the true Euler angles $\rho \in\{\alpha, \beta, \gamma\}$ and the estimated ones $\tilde{\rho}$ (in degrees) for the Noisy Rotations and Noisy Measurements scenarios, respectively. The results in Table 1 indicate that the discrepancy decreases as the amount of error in rotation, $\sigma_{\text {rot }}$, decreases. In particular, we achieve very low discrepancies (less than $3.3^{\circ}$ ) for all $\sigma_{\text {rot }} \geq \frac{\pi}{45}$. We also observe that the number of correspondences, $N$, does not have a significant effect on the estimation of the irreducible representation in (10). The results in Table 2 show a similar decreasing trend in $\delta$ when the SNR increases. This time, an increase in the number of correspondences also reduces the angular discrepancies, which are less than $2.7^{\circ}$ for all SNRs when $N \geq 40$. This demonstrates that the solution of (10) becomes more reliable when $N$ increases.

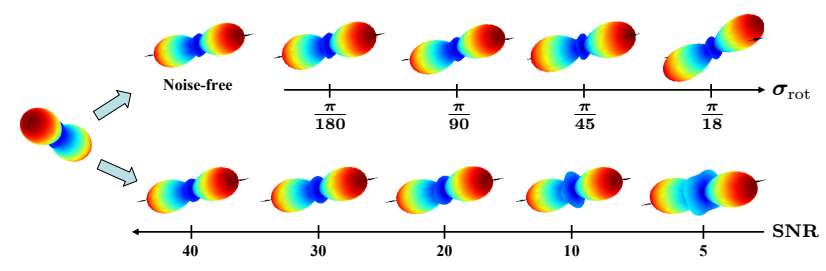

Fig. 1. Generation of ODF correspondences for the sensitivity experiment: The ODFs are color-coded (blue low, red high) and the black lines with arrowheads indicate the correct ODF maxima direction after rotation in the absence of noise.

\subsection{Experiments on Phantom Data}

We use the proposed approach for aligning two ODF images that are assumed to be related via a rigid body transformation, i.e., rotation $\mathbf{R}$ and translation $\mathbf{t}$ such that $\mathbf{x}_{2}=\mathbf{R} \mathbf{x}_{1}+\mathbf{t}$. This experiment can be considered as a simulation of correcting for subject motion. We use a realistic diffusion MR phantom [15] mimicking the diffusion properties of white matter. Fig. 2(a) shows the configuration of the fiber tracts in the phantom. The HARDI signal is acquired with a $64 \times 64$ image matrix, an isotropic spatial resolution of $3 \mathrm{~mm}$, and a diffusion sensitization at $b=1,500 \mathrm{~s} / \mathrm{mm}^{2}$ applied along a set of 64 gradient directions. The reconstructed ODFs are shown in Fig. 2(b).

We apply different rigid body transformations $\left\{\left(\mathbf{R}_{j}, \mathbf{t}_{j}\right)\right\}$ to the source image to obtain the target image. The rotations are of the form $\mathbf{R}(\alpha, 0,0)$ with $\alpha \in\left\{0, \frac{\pi}{12}, \frac{\pi}{6}, \ldots, \frac{\pi}{2}\right\}$. Once the angle $\alpha$ is chosen, we add Gaussian noise with zero mean and standard deviation $\sigma_{\text {rot }}=$ $\frac{\pi}{90}$ to obtain the rotations $\left\{\mathbf{R}_{j}\right\}$. The experiment is repeated 10 times with random translations $\left\{\mathbf{t}_{j}=\left[t_{x}, t_{y}, t_{z}\right]^{\top}\right\}$, where $\left[t_{x}, t_{y}\right]^{\top} \sim$ $\mathcal{N}\left(\mathbf{0}, 4 \mathbf{I}_{2 \times 2}\right)$ and $t_{z}=0$. After transforming the ODF fields, the correspondences are established by matching the SIFT [16] features between the source and target generalized fractional anisotropy (GFA) maps [1]. This procedure provides a number of ODF correspondences $\left\{\left(p_{1 n}, p_{2 n}\right)\right\}$ and point correspondences $\left\{\left(\mathbf{x}_{1 n}, \mathbf{x}_{2 n}\right)\right\}$ for estimating the spatial transformation, as shown in Figs. 2(c) and 2(d) when $\mathbf{R} \equiv$ $\mathbf{R}\left(\frac{\pi}{2}, 0,0\right)$. We then apply the proposed approach to estimate the rotation, reorient the ODFs and then use the point correspondences to find the translation via least-squares. The mean and standard deviation of $\delta_{\alpha}, \delta_{\beta}$, and $\delta_{\mathbf{t}}=\|\tilde{\mathbf{t}}-\mathbf{t}\|_{2}$ is calculated as $2.66 \pm 2.95^{\circ}, 4.26 \pm$ $3.44^{\circ}$ and $0.96 \pm 0.45$ pixels, respectively. The errors in $\alpha$ and $\mathbf{t}$ are relatively small, which demonstrates that our approach yields accurate initial information to resolve simple parametric transformations.

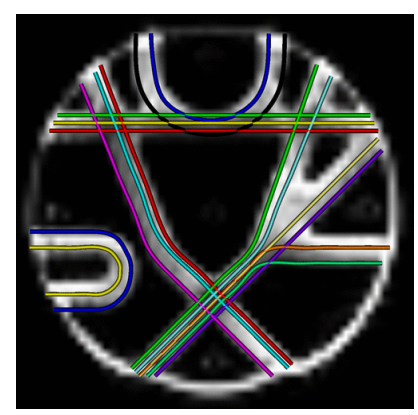

(a)

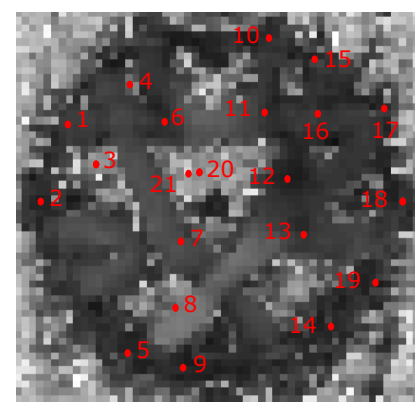

(c)

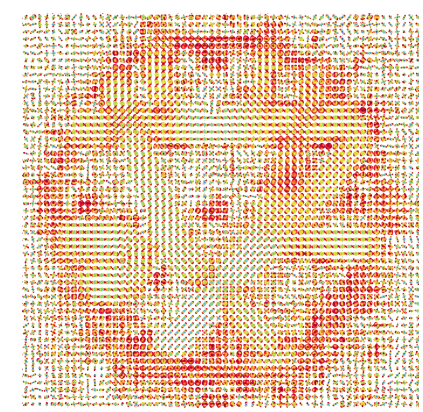

(b)

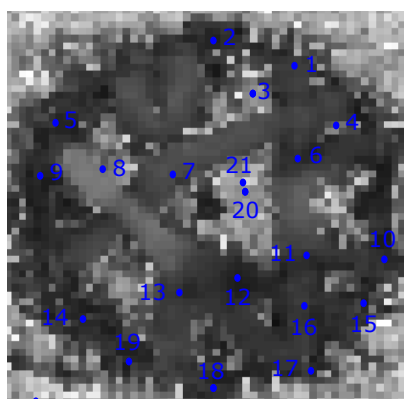

(d)
Fig. 2. (a) The fiber tract configuration in the phantom, (b) The reconstructed ODFs. Automatically identified ODF/point correspondences between (c) the GFA map of the source ODFs and (d) the GFA map of the target ODFs rotated $\left(\frac{\pi}{2}, 0,0\right)$ radians. 
Table 1. Averages of the angular discrepancies $\left\{\delta_{\alpha}, \delta_{\beta}, \delta_{\gamma}\right\}$ (in degrees) in the Noisy Rotations scenario.

\begin{tabular}{|c|c|c|c|c|c|c|c|c|c|c|c|c|c|c|c|}
\hline \multirow[b]{2}{*}{$\sigma_{\text {rot }}$} & \multicolumn{3}{|c|}{$N=20$} & \multicolumn{3}{|c|}{$N=40$} & \multicolumn{3}{|c|}{$N=60$} & \multicolumn{3}{|c|}{$N=80$} & \multicolumn{3}{|c|}{$N=100$} \\
\hline & $\delta_{\alpha}$ & $\delta_{\beta}$ & $\delta_{\gamma}$ & $\delta_{\alpha}$ & $\delta_{\beta}$ & $\delta_{\gamma}$ & $\delta_{\alpha}$ & $\delta_{\beta}$ & $\delta_{\gamma}$ & $\delta_{\alpha}$ & $\delta_{\beta}$ & $\delta_{\gamma}$ & $\delta_{\alpha}$ & $\delta_{\beta}$ & $\delta_{\gamma}$ \\
\hline$\pi / 18$ & 9.43 & 6.86 & 7.98 & 9.47 & 6.83 & 7.97 & 9.45 & 6.81 & 7.99 & 9.50 & 6.77 & 8.01 & 9.45 & 6.78 & 8.00 \\
\hline$\pi / 45$ & 3.14 & 2.32 & 3.25 & 3.24 & 2.32 & 3.24 & 3.13 & 2.33 & 3.20 & 3.11 & 2.34 & 3.26 & 3.10 & 2.31 & 3.23 \\
\hline$\pi / 90$ & 1.39 & 1.42 & 1.54 & 1.40 & 1.42 & 1.55 & 1.40 & 1.42 & 1.54 & 1.39 & 1.42 & 1.57 & 1.38 & 1.43 & 1.57 \\
\hline$\pi / 180$ & 0.73 & 0.90 & 0.68 & 0.73 & 0.90 & 0.66 & 0.72 & 0.90 & 0.67 & 0.73 & 0.90 & 0.66 & 0.73 & 0.89 & 0.68 \\
\hline
\end{tabular}

Table 2. Averages of the angular discrepancies $\left\{\delta_{\alpha}, \delta_{\beta}, \delta_{\gamma}\right\}$ (in degrees) in the Noisy Measurements scenario.

\begin{tabular}{|c|c|c|c|c|c|c|c|c|c|c|c|c|c|c|c|}
\hline \multirow{2}{*}{ SNR } & \multicolumn{3}{|c|}{$N=20$} & \multicolumn{3}{|c|}{$N=40$} & \multicolumn{3}{|c|}{$N=60$} & \multicolumn{3}{|c|}{$N=80$} & \multicolumn{3}{|c|}{$N=100$} \\
\hline & $\delta_{\alpha}$ & $\delta_{\beta}$ & $\delta_{\gamma}$ & $\delta_{\alpha}$ & $\delta_{\beta}$ & $\delta_{\gamma}$ & $\delta_{\alpha}$ & $\delta_{\beta}$ & $\delta_{\gamma}$ & $\delta_{\alpha}$ & $\delta_{\beta}$ & $\delta_{\gamma}$ & $\delta_{\alpha}$ & $\delta_{\beta}$ & $\delta_{\gamma}$ \\
\hline 5 & 6.53 & 2.11 & 6.92 & 2.04 & 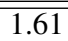 & 2.62 & 2.12 & 1.55 & 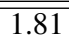 & 1.30 & 1.31 & 1.22 & 1.23 & 1.25 & 1.11 \\
\hline 10 & 4.04 & 1.86 & 4.25 & 2.28 & 1.53 & 2.12 & 0.90 & 1.18 & 0.95 & 0.91 & 0.91 & 0.84 & 0.80 & 1.06 & 0.64 \\
\hline 20 & 1.57 & 1.12 & 1.22 & 1.32 & 1.02 & 0.80 & 0.89 & 0.81 & 0.50 & 0.71 & 0.71 & 0.43 & 0.47 & 0.74 & 0.42 \\
\hline 30 & 1.58 & 1.07 & 1.84 & 1.57 & 0.90 & 0.58 & 1.13 & 0.87 & 0.29 & 0.42 & 0.71 & 0.22 & 0.37 & 0.82 & 0.29 \\
\hline 40 & 1.40 & 1.14 & 1.79 & 1.34 & 0.86 & 0.52 & 0.67 & 0.78 & 0.43 & 0.45 & 0.68 & 0.22 & 0.22 & 0.62 & 0.18 \\
\hline
\end{tabular}

\section{DISCUSSIONS AND CONCLUSIONS}

We proposed an algebraic solution to the problem of estimating the rotation aligning multiple pairs of ODFs. These pairs (or correspondences) were used to compute the linear transformation between the $\mathrm{SH}$ coefficients of the ODFs, which is an irreducible unitary representation of the rotation. Our formulation provides a unique solution that can be obtained in closed form. The Euler angles parameterizing the rotation were computed from the elements of this representation in a sequential way, which reduces the sensitivity to initialization. We demonstrated that this strategy yields accurate estimates of the rotation parameters and offers robustness to moderate amounts of noise.

To generate the $\mathrm{ODF} /$ point correspondences, a medical expert can manually provide landmarks that correspond to the same anatomical structure in the target and source images. However, this procedure may be challenging, time consuming, and error-prone. To alleviate this problem to some extent, we adopted an automatic strategy based on feature extraction and matching, and showed its applicability in our experiments on phantom data. More specifically, we used SIFT features, which measure the distribution of gradients around a pixel and are invariant to image translation, rotation and scaling. The similarity between the features was measured in terms of a matching score and the number of correspondences can be altered by changing the value of the threshold for this score. It is worth noting that this type of automatic strategies might also yield incorrect/inadequate number of correspondences and user intervention might still be needed.

While registering HARDI data characterized by ODFs, one should always consider the fact that a spatial transformation induces a reorientation of the ODF because the direction of diffusion depends on the coordinate system. The proposed approach can be considered as a method that estimates a "global reorientation function" from ODF correspondences without knowing the deformation. Although this assumes a highly oversimplified model to capture inter-subject variability of human brain, our formulation is suitable for featurebased intra-subject alignment, e.g., correction for subject motion, which is a possible direction for future work. In addition, further research is needed to formulate the problem using quaternions so that one can avoid singularities when working with the Euler angles.

\section{REFERENCES}

[1] D.S. Tuch, "Q-ball imaging," Magnetic Resonance in Medicine, vol. 52 , no. 6, pp. 1358-1372, 2004.
[2] P.J. Basser, J. Mattiello, and D. LeBihan, "Estimation of the effective self-diffusion tensor from the NMR spin echo," Journal of Magnetic Resonance B, vol. 103, no. 3, pp. 247-254, 1994.

[3] P.-T. Yap, Y. Chen, H. An, Y. Yang, J.H. Gilmore, W. Lin, and D. Shen, "SPHERE: Spherical harmonic elastic registration of HARDI data," NeuroImage, vol. 55, no. 2, pp. 545-556, 2011.

[4] L. Bloy and R. Verma, "Demons registration of high angular resolution diffusion images," in IEEE Int. Symposium on Biomedical Imaging, 2010, pp. 1013-1016.

[5] X. Geng, T.J. Ross, H. Gu, W. Shin, W. Zhan, Y.-P. Chao, C.-P. Lin, N. Schuff, and Y. Yang, "Diffeomorphic image registration of diffusion MRI using spherical harmonics," IEEE Transactions on Medical Imaging, vol. 30, no. 3, pp. 747-758, 2011.

[6] D. Raffelt, J-D. Tournier, J. Fripp, S. Crozier, A. Connelly, and O. Salvado, "Symmetric diffeomorphic registration of fibre orientation distributions," NeuroImage, vol. 56, no. 3, pp. 1171-1180, 2011.

[7] J. Du, A. Goh, and A. Qiu, "Large deformation diffeomorphic metric mapping of orientation distribution functions," in Information Processing in Medical Imaging, 2011, LNCS 6801/2011, pp. 448-462.

[8] A. Makadia and K. Daniilidis, "Direct 3D-rotation estimation from spherical images via a generalized shift theorem," in IEEE Conference on Computer Vision and Pattern Recognition, 2003, pp. 217-224.

[9] A. Makadia and K. Daniilidis, "Rotation recovery from spherical images without correspondences," IEEE Transactions on Pattern Analysis and Machine Intelligence, vol. 28, no. 7, pp. 1170-1175, 2006.

[10] M. Descoteaux, E. Angelino, S. Fitzgibbons, and R. Deriche, "Regularized, fast and robust analytical Q-ball imaging," Magnetic Resonance in Medicine, vol. 58, no. 3, pp. 497-510, 2007.

[11] G.S. Chirikjian and A.B. Kyatkin, Engineering Applications of Noncommutative Harmonic Analysis: With Emphasis on Rotation and Motion Groups, CRC Press, 2000.

[12] Z. Su and P. Coppens, "Rotation of real spherical harmonics," Foundations of Crystallography, vol. A50, no. 5, pp. 636-643, 1994.

[13] R. Horn and C.R. Johnson, Matrix Analysis, Cambridge University Press, 1985.

[14] A. Goh, C. Lenglet, P.M. Thompson, and R. Vidal, "Estimating orientation distribution functions with probability density constraints and spatial regularity," in Medical Image Computing and Computer-Assisted Intervention, 2009, vol. LNCS 5761, pp. 877-885.

[15] C. Poupon, B. Rieul, I. Kezele, M. Perrin, F. Poupon, and J.-F. Mangin, "New diffusion phantoms dedicated to the study and validation of high-angular-resolution diffusion imaging (HARDI) models," Magnetic Resonance in Medicine, vol. 60, no. 6, pp. 1276-1283, 2008.

[16] D. Lowe, "Distinctive image features from scale-invariant keypoints," Int. Journal of Computer Vision, vol. 20, pp. 91-110, 2004. 